\title{
The Prefiltering Techniques in Emotion Based Place Recommendation Derived by User Reviews
}

\author{
U. A. Piumi Ishanka and Takashi Yukawa \\ Graduate School of Engineering, Nagaoka University of Technology, 1603-1 Kamitomioka-machi, Nagaoka, Japan \\ Correspondence should be addressed to U. A. Piumi Ishanka; s135063@stn.nagaokaut.ac.jp
}

Received 2 August 2017; Accepted 19 September 2017; Published 22 October 2017

Academic Editor: Shyi-Ming Chen

Copyright ( 2017 U. A. Piumi Ishanka and Takashi Yukawa. This is an open access article distributed under the Creative Commons Attribution License, which permits unrestricted use, distribution, and reproduction in any medium, provided the original work is properly cited.

\begin{abstract}
Context-aware recommendation systems attempt to address the challenge of identifying products or items that have the greatest chance of meeting user requirements by adapting to current contextual information. Many such systems have been developed in domains such as movies, books, and music, and emotion is a contextual parameter that has already been used in those fields. This paper focuses on the use of emotion as a contextual parameter in a tourist destination recommendation system. We developed a new corpus that incorporates the emotion parameter by employing semantic analysis techniques for destination recommendation. We review the effectiveness of incorporating emotion in a recommendation process using prefiltering techniques and show that the use of emotion as a contextual parameter for location recommendation in conjunction with collaborative filtering increases user satisfaction.
\end{abstract}

\section{Introduction}

Context-awareness has been introduced into recommendation systems to ensure that both long-term and short-term user needs are recognized by considering not only preference history but also the current situation. This is because if the recommendation process only relies on preference history, it may not correctly discard an isolated situation such as a gift purchase (i.e., if a user buys a gift for a friend's child, a baby suit, user receives suggestions for the baby items repeatedly) because it cannot adapt to the current situation. Context-aware recommendation systems (CARS) therefore incorporate contextual information including location, time, and activity and even advanced parameters such as emotion and personality.

Previous studies of recommendation systems have investigated the incorporation of emotion into the recommendation process with a particular emphasis on the impact of emotion and personality traits on the human decisionmaking process. The role of emotion has been identified in different stages of the content consumption process including (i) the entry stage, (ii) the consumption stage, and (iii) the exit stage, while the consumption stage is highly influenced by emotion [1]. Emotions are mental states usually caused by an event of importance to the subject and have been modeled in various studies. The universal model classifies emotion into fixed categories such as Ekman's six basic emotions (happiness, anger, fear, sadness, disgust, and surprise) [2] and Plutchik's eight basic and prototypical emotions (joy, sadness, anger, fear, trust, disgust, surprise, and anticipation) [3]. In the dimensional model, each emotion is described as a point in a continuous multidimensional space, where each dimension represents a quality of an emotion. The most frequently used dimensions are valence, arousal, and dominance [1].

According to various psychological studies, a place can have an impact on memories, sentiments, and emotional well-being [4]. Debored [5] introduced the concept of psychogeography and tracked the influence of geographical environments on the emotions and behavior of individuals. Although location is commonly analyzed by considering context and its impact on emotional well-being, no taxonomy has been derived for the emotion that a location may evoke [6]. Moreover, it is vital that any dataset contains affective parameters. Studies of music and movie recommendation systems datasets have used the LDOS-CoMoDa, LJ2M, myPersonality, LDOS-PerAff-1, DEAP, ANET, IADS, ANEW, 


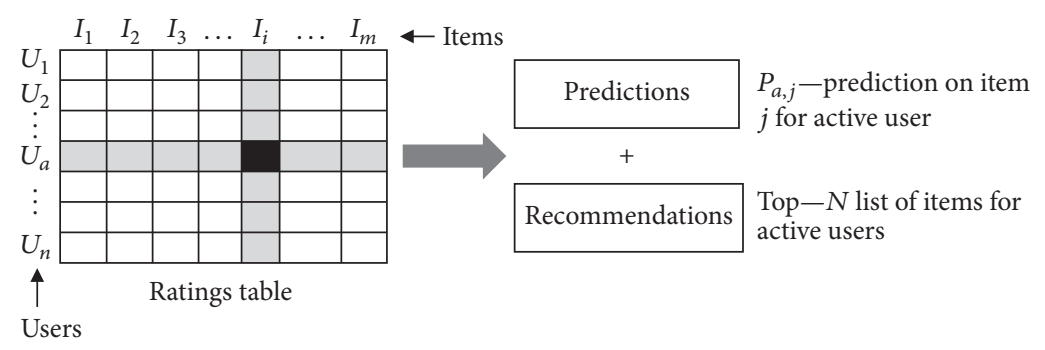

FIGURE 1: Collaborative filtering process.

IAPS, and 1000 songs datasets [7], but none of these relate to tourist destination recommendation data.

In development of the proposed system, we addressed the paucity of datasets with contextual parameters by employing Sentimental Analysis (SA) to acquire the emotional states of users based on user reviews for 100 destinations. We then derived emotional tags and manually compared the accuracy of the tags for each of the destinations.

In this study, a CARS that uses partial contextual user preferences in the form of user, item, context, and rating was developed. This is unlike a traditional recommendation system, which is based on knowledge of preferences of a set of items and the input data is in the form of user, item, and rating. The contextual information that we consider here can be applied to various stages of the recommendation process. A CAR process based on contextual user preferences elicitation and estimation can take one of three forms as follows: contextual prefiltering, contextual postfiltering, or contextual modeling. In this study, we implemented a contextual prefiltering paradigm-based solution and use the contextual information to select the most relevant item $\times$ user data for generating recommendations [8]. The prefiltering approach allows for the deployment of any of the numerous traditional recommendation techniques previously proposed [9].

The main objectives of this study are as follows:

(1) Deriving suitable datasets for tourist destination recommendations considering emotion as a contextual parameter

(2) Examining how emotion influences recommendation of destinations using prefiltering techniques.

\section{Background}

The body of literature related to recommendation systems is constantly expanding. Previous studies have introduced algorithms for recommendations, improved ways of building user models to represent user preferences, interests, and behaviors, and domain-specific applications [10]. Petrevska and Koceski (2012) proposed a tourism recommendation system based on user preferences, interests, and desires and suggested tourist attractions based on profiling user behaviors such as reading other reviews to make decisions [11]. Destinations were observed as objects and changes in user preferences following subsequent visits tracked according to the rating and user behavior was considered in the profiling and recommendations generated based on collaborative filtering. Sarkaleh et al. (2012) suggested a tourism recommendation system using location and user features as contextual parameters [12]. Zheng et al. (2012) proposed a CARS for travel recommendations using differential contextual relaxation and considered trip type, duration, originality, destination city, and month as contextual parameters with user collaborative filtering to propose a hybrid recommendation to mitigate the disadvantages of the solo use of prefiltering and postfiltering techniques [13]. De Pessemier et al. (2015) suggested a system with group recommendation for traveler destinations based on a user's rating profile, personal interests, and specification for their next destination by following a hybrid approach in combination with content-based, collaborative filtering, and knowledge-based strategies [14]. Collaborative filtering is a widely adapted recommendation algorithm in which predictions and recommendations are based on the ratings or the behavior of the users in the system $[15,16]$.

The approach considers a list of $n$ number of users $U$ and list of $m$ number of items $I$. As shown in Figure 1, the user $u_{a}, u_{a} \in U$ is called the active user for whom the task of the collaborative filtering algorithm is to find an item likeliness that can be in two forms. $P_{a, j}$ is the predictive rating for item $i$ for the active user $U_{a}$, where $j \in m$. The prediction rating is a numerical value with the same scale as the rating provided by user $u_{a}$. The recommendation is the list of $N$ items that the active user will like the most [17].

Collaborative filtering techniques can be either userbased or item-based. The user-based collaborative filtering model recommends items based on computing similar neighbors and creates a group of users that are compatible with a target user. Item-based collaborative filtering computes similarity based on items and finds items that are similar to the given user's rated items [18]. A traditional recommendation system starts by estimating the rating function $\mathbf{R}$ using an initialized set of ratings that are either explicitly provided by users or are implicitly inferred by the system for user $u$ and item $i$ that has not been rated.

$$
\mathbf{R}: \text { user } \times \text { item } \longrightarrow \text { rating. }
$$

In a CARS, preferences are predicted by incorporating available contextual information into the recommendation process and $\mathbf{R}$ is estimated by

$$
\text { R: user } \times \text { item } \times \text { context } \longrightarrow \text { rating, }
$$

where user and item are the space of users and items and rating is the space of rating for the user and item pairs. The 


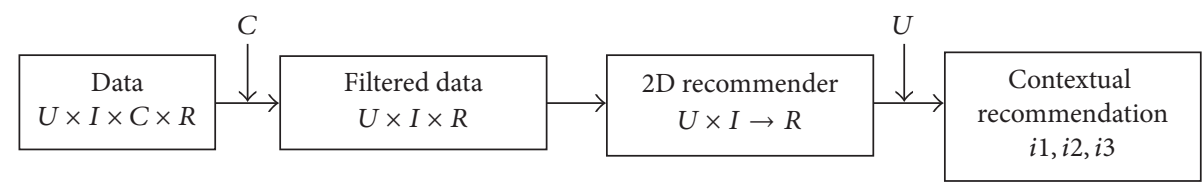

FIGURE 2: Prefiltering process.

contextual information can be applied during various stages of the recommendation process and the form of the contextaware recommendation can be contextual prefiltering (contextualization of input), contextual postfiltering (contextualization of output), or contextual modeling (contextualization of recommendation function) [8]. In contextual prefiltering, the contextual information is used to select the relevant set of ratings, and ratings are predicted for active users using any traditional recommendation function. The context is then set as a query for filtering relevant ratings (see Figure 2).

The most appropriate use of prefiltering technique can vary with the application. One approach is to use a model that targets a local context model for each situation. Another is to use generalized prefiltering, which allows for the generalization of the data filtering query based on a specific context [19]. Generalized prefiltering states the ratings based on the related contextual situations and derives a collection of prediction models based upon the ratings for each segment.

Affective computing and SA are combined in various research fields and many of advanced SA techniques have been developed and many commercial and academic tools, such as IBM (https://www.ibm.com/analytics), SAS (https:// www.sas.com/social), Oracle (https://www.oracle.com/social), SenticNet (http://www.business.sentic.net), and Luminoso (https://www.luminoso.com) emerged for facilitating polarity evaluations and or mood classification, though most of them are costly and highly limited to set of emotions [20]. SA is considered as computational treatment of opinions, sentiments, and subjectivity of texts, while opinion mining is a tedious task, since a comprehensive knowledge of most of the explicit and implicit, regular and irregular, and syntactic and semantic rules of a language is to be considered in the process $[21,22]$.

In the state of the art of sentiment classification techniques, the lexicon approaches rely on a sentiment lexicon and the classifications methods using machine learning can be further divided into supervised and unsupervised learning methods. The supervised learning methods comprised many classifiers including probabilistic classifier, naive Bayes classifier, linear classifier, Support Vector Machine classifiers, Neural Network, decision tree classifiers, rule-based classifiers, and meta-based classifiers [21]. Currently the SA techniques are enriched with deep learning approaches like Deep Convolutional Neural Networks as well. The Convolutional Neural Networks (CNNs) are very similar to ordinary Neural Networks (NN); the main difference is the number of layers where CNN are just several layers of convolutions with nonlinear activation functions applied to the results, while in traditional $\mathrm{NN}$, each input neuron is connected to each output neuron in the next layer. In CNNs, instead, convolutions are used over the input layer to compute the output. This results in local connections, where each region of the input is connected to a neuron in the output. Each layer applies different filters, typically hundreds or thousands, and combines their results $[23,24]$. A drawback of $\mathrm{CNN}$ as a classifier is that it finds only a local optimum, since it uses the same backpropagation technique as MLP [25].

\section{Incorporating Emotion into Place Recommendation}

3.1. Emotion Tag Acquisition. The main challenge with a CARS domain is the lack of appropriate contextual datasets. The LDOS-PerAff- 1 corpus is one of the datasets created to fulfill this issue [26]. It incorporates video clips of users responding to emotional stimuli and ratings with personality traits. Data acquisition was performed by presenting a set of images and asking subjects to rate the images as if they were choosing images for their computer wallpaper. The LDOS-CoMoDa corpus is another dataset introduced as a context-aware movie dataset comprising 12 contextual dimensions with 2291 ratings rated by 121 users on 1232 items. Among the contextual parameters suggested, three emotional dimensions were included; endEmo, emotional states at the end of the movie; domEmo, the emotional state experienced most during the movie; and mood, the emotion of the user, when they are watching the movie [27]. The acquisition of emotion has been investigated using a variety of technologies such as the detection of facial expressions, emotion inference from sensors, and other approaches based on voice, speech, body language, and postures. The difficulty in applying these techniques in the recommendation system domain is the complexity in adapting these techniques to the system implementation. Therefore, previous studies have focused on emotion states inferred from reviews by using SA theory. SA techniques can be used to extract emotion from review texts including joy, sadness, fear, anger, and surprise [21]. Emotion detection can be implemented using machine learning or lexicon-based approaches, with the latter being more frequently used [27].

In this study, a context-aware dataset was derived by collecting data for the global top 100 tourist attractions in 2016. The data including description, location, and images were obtained from Wikipedia (https://en.wikipedia.org/), while the average rating and 100 user reviews for each destination were collected from TripAdvisor (https://www.tripadvisor .com/). The reviews were analyzed and classified to acquire emotion tags to represent user emotional states for a place in two stages. First, we used Tone Analyzer (https://tone-analyzerdemo.mybluemix.net/), which measures emotional tone, to get a sense of the overall tone of the review (joy, fear, sadness, disgust, and anger). Second, we expanded the positive 
TABLE 1: Sample results of tone analysis created by Tone Analyzer.

\begin{tabular}{|c|c|c|c|c|c|}
\hline Place ID & Anger & Disgust & Fear & Joy & Sadness \\
\hline 10 & 0.073451 & 0.114556 & 0.111547 & 0.530747 & 0.234107 \\
\hline 11 & 0.074452 & 0.115561 & 0.109812 & 0.501336 & 0.259509 \\
\hline 12 & 0.082424 & 0.065996 & 0.063544 & 0.617 & 0.265856 \\
\hline 13 & 0.091267 & 0.115791 & 0.120392 & 0.506598 & 0.231926 \\
\hline 14 & 0.151867 & 0.076331 & 0.070043 & 0.567042 & 0.267992 \\
\hline 15 & 0.135269 & 0.064383 & 0.101094 & 0.545255 & 0.260857 \\
\hline 16 & 0.142556 & 0.161189 & 0.165065 & 0.422934 & 0.319239 \\
\hline 17 & 0.095104 & 0.06126 & 0.152121 & 0.513339 & 0.264931 \\
\hline 18 & 0.123192 & 0.102279 & 0.113161 & 0.524549 & 0.360327 \\
\hline 19 & 0.092798 & 0.086266 & 0.113484 & 0.526654 & 0.240624 \\
\hline 20 & 0.067278 & 0.072824 & 0.074158 & 0.611672 & 0.285493 \\
\hline
\end{tabular}

TABLE 2: Extract from the emotion lexicon used in the classification.

\begin{tabular}{|c|c|c|c|c|c|c|c|}
\hline Joy & Surprise & Anticipation & Trust & Fear & Anger & Sadness & Disgust \\
\hline Absolution & Abandonment & Abundance & Abacus & Abandon & Abandoned & Abandon & Aberration \\
\hline Abundance & Abduction & Accelerate & Abbot & Abandoned & Abandonment & Abandoned & Abhor \\
\hline Abundant & Abrupt & Accolade & Absolution & Abandonment & Abhor & Abandonment & Abhorrent \\
\hline Accolade & Accident & Accompaniment & Abundance & Abduction & Abhorrent & Abduction & Abject \\
\hline Accompaniment & Accidental & Achievement & Academic & Abhor & Abolish & Abortion & Abnormal \\
\hline Accomplish & Accidentally & Acquiring & Accolade & Abhorrent & Abomination & Abortive & Abominable \\
\hline Accomplished & Accolade & Addresses & Accompaniment & Abominable & Abuse & Abscess & Abomination \\
\hline Achieve & Advance & Adore & Accord & Abomination & Accursed & Absence & Abortion \\
\hline Achievement & Affront & Adrift & Account & Abortion & Accusation & Absent & Abundance \\
\hline Acrobat & Aghast & Advance & Accountability & Absence & Accused & Absentee & Abuse \\
\hline
\end{tabular}

emotion scale from "joy" to "joy, anticipation, trust, and surprise" and employed SA techniques. Text preprocessing techniques were used to derive emotion tags from reviews for each location. A list of words indicative of each emotion was used. The emotion lexicon from the National Research Council (NRC), Canada, which is based on Plutchik's eight emotions, and two negative and positive sentiments [28] were used to calculate term frequencies.

The lexicon set derived by NRC comprises unigrams and bigrams of the Macquarie Thesaurus [29], all terms in the General Inquirer, and the WordNet-Affect Lexicon. Thus, the used lexicon set comprises unigrams and bigrams of the Macquarie Thesaurus: 800 and 787 lexicons as adjectives, adverbs, nouns, and verbs, respectively; all the terms in the General Inquirer: 8132 lexicons as negative, positive, and neutral; and the WordNet-Affect Lexicon: 640 lexicons representing emotion categories anger, disgust, fear, joy, surprise, and sadness.

In the Tone Analyzer analysis, for each emotion, a score of less than 0.5 indicates that the emotion is unlikely to be perceived in the content and a score greater than 0.75 indicates a high likelihood that the emotion will be perceived. The overall results show that the highest tone value was recorded in the joy group for all locations, and $88 \%$ of the joy group indicates that the emotion is likely to be perceived in the selected review texts (see Table 1).
Text preprocessing is a significant task in text mining techniques, and its application is the first step in any system. The main aim behind it is to represent each document as a feature vector, so it separates the text into individual words. The quality of the classification process is highly dependent on this feature selection process. Therefore, it is important to select meaningful keywords and discard words that do not enable distinguishing of the documents [30].

Next, we performed a term frequencies (TFs) calculation based on the lexicons of the eight emotion groups. Table 2 illustrates the part of the lexicon used in classification. We chose the emotion category for a destination based upon the maximum frequency value as shown in Table 3. The TFs were calculated for each review and the total TFs were calculated for each emotion. We then selected the emotion tag for each destination based on the highest frequency (see Table 4).

In the case of the occurrence of multiple emotions, we assumed that if a negative emotion category and a positive emotion category appeared with equal frequency, the negative one was stronger. This assumption is based on the fact that humans are more honest when stating negative emotions. In terms of the positive emotions occurring with an equal frequency, we assumed that joy, trust, anticipation, and surprise are stated in a descending order by considering how humans tend to identify and state these emotions (see Table 4). 
TABLE 3: Emotion tag derivation based on TF values.

\begin{tabular}{|c|c|c|c|c|c|c|c|c|c|}
\hline Place ID & User ID & An & Ant & $\mathrm{D}$ & $\mathrm{F}$ & $\mathrm{J}$ & $\mathrm{S}$ & $\mathrm{Su}$ & $\mathrm{T}$ \\
\hline 1 & 1 & 1 & 3 & 1 & 0 & 4 & 0 & 2 & 3 \\
\hline 1 & 2 & 0 & 1 & 0 & 0 & 0 & 0 & 0 & 1 \\
\hline 1 & 3 & 1 & 3 & 1 & 1 & 3 & 2 & 2 & 2 \\
\hline 1 & 4 & 1 & 0 & 1 & 0 & 1 & 0 & 0 & 1 \\
\hline \multirow[t]{2}{*}{1} & 5 & 1 & 3 & 0 & 2 & 1 & 1 & 1 & 1 \\
\hline & Emotion word sum & 4 & 10 & 3 & 3 & 9 & 3 & 5 & 8 \\
\hline 1 & Emotion tag & \multicolumn{8}{|c|}{ Anticipation } \\
\hline
\end{tabular}

An: angry; Ant: anticipation; D: disgust; F: fear; J: joy; S: sadness; Su: surprise; and T: trust.

TABLE 4: Emotion tag derivation of TFs.

\begin{tabular}{|c|c|c|c|c|c|c|c|c|c|}
\hline Place ID & $\mathrm{J}$ & $\mathrm{Su}$ & $\mathrm{T}$ & Ant & $\mathrm{D}$ & $\mathrm{F}$ & An & $S$ & Emotion tag \\
\hline 1 & 29 & 14 & 55 & 42 & 6 & 12 & 13 & 16 & Trust \\
\hline 2 & 28 & 12 & 32 & 23 & 1 & 10 & 5 & 4 & Trust \\
\hline 3 & 31 & 14 & 21 & 24 & 0 & 3 & 4 & 10 & Joy \\
\hline 4 & 17 & 7 & 17 & 13 & 0 & 6 & 4 & 6 & Joy \\
\hline 5 & 39 & 18 & 44 & 44 & 5 & 14 & 10 & 11 & Trust \\
\hline 6 & 31 & 32 & 43 & 41 & 8 & 11 & 16 & 18 & Trust \\
\hline 7 & 51 & 26 & 41 & 38 & 2 & 3 & 2 & 8 & Joy \\
\hline 8 & 46 & 17 & 48 & 39 & 9 & 11 & 10 & 9 & Trust \\
\hline 9 & 24 & 13 & 22 & 22 & 2 & 8 & 6 & 11 & Joy \\
\hline 10 & 23 & 11 & 28 & 21 & 1 & 6 & 4 & 5 & Trust \\
\hline 11 & 45 & 19 & 49 & 36 & 5 & 12 & 4 & 7 & Trust \\
\hline 12 & 44 & 31 & 26 & 64 & 4 & 7 & 7 & 5 & Anticipation \\
\hline 13 & 18 & 7 & 20 & 16 & 1 & 5 & 4 & 4 & Trust \\
\hline 14 & 49 & 21 & 47 & 62 & 6 & 14 & 7 & 9 & Anticipation \\
\hline 15 & 35 & 16 & 37 & 43 & 2 & 21 & 7 & 12 & Anticipation \\
\hline
\end{tabular}

An: angry; Ant: anticipation; D: disgust; F: fear; J: joy; S: sadness; Su: surprise; and T: trust.

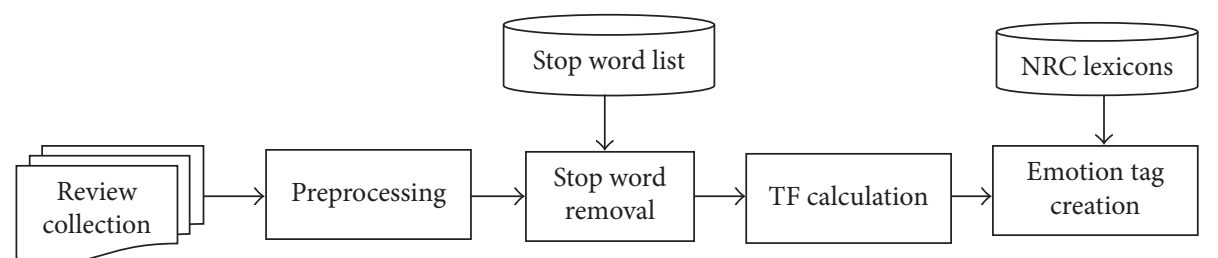

FIgURE 3: Emotion tag creation.

We collected 9998 ratings from 8470 users for the 100 selected locations and derived emotion tags for each location for each user. From this information, we derived two datasets: place dataset \{place id, name, description, place category, location, average rating, emotion tag\} and user dataset $\{$ user $i d$, place id, user emotion tag, user rating . Figure 3 illustrates the emotion tag derivation process.

$A$ is the total number of cases (destinations) to which the system assigned emotion tags.

$C$ is the number of correct cases based on manual judgment.

$$
\text { Precision }=\frac{C}{A}
$$

We calculated the precision of emotion tag detection and compared the results manually by reading review texts for the place dataset. The results show that the precision of the detection process is $63.3 \%$ [31].

3.2. Recommendation System Development. We implemented the proposed recommendation system using the two derived datasets and loaded the location data into the database of the system. The dataset was input to the recommendation function based on the emotion state of the user when they logged in, as shown in Figure 4. Using the prefiltering techniques in CARS, the similarity and predictive rating values were calculated for each user and the top five place 


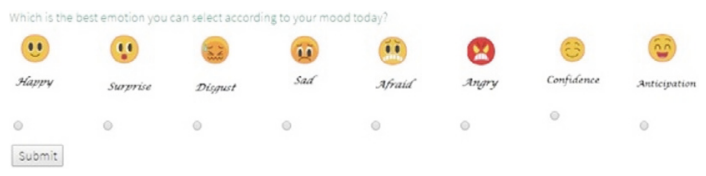

Figure 4: Emotion gathering.

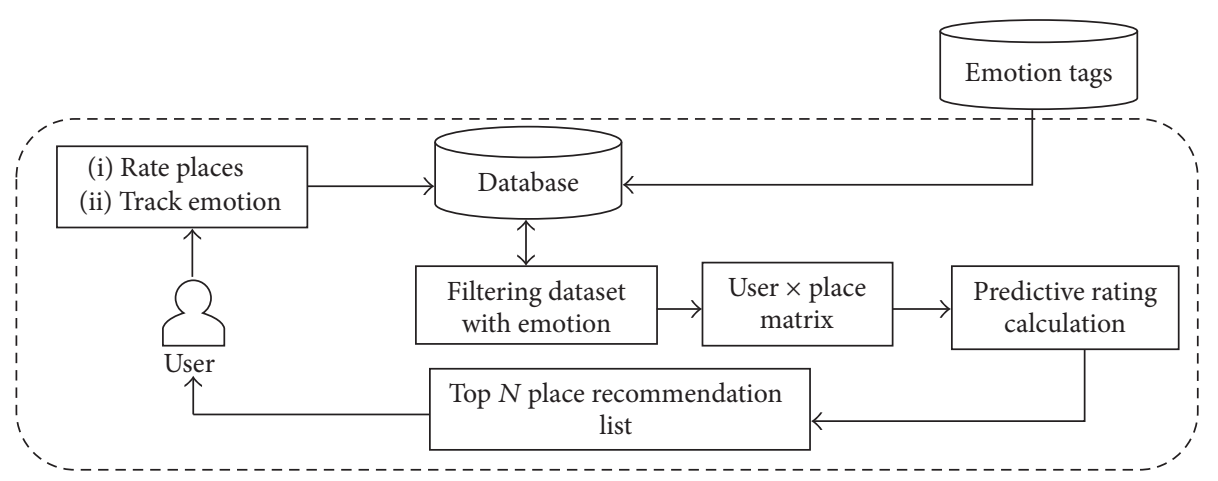

FIGURE 5: Recommendation process.

recommendations were generated. The system's recommendation engine consists of two phases based on collaborative filtering without emotion (CFN) and collaborative filtering with emotion (CFE).

In the implementation, we used item-item collaborative filtering to develop and review our contextual parameters on the derived dataset. The recommendation process is illustrated in Figure 5.

Each recommendation based on emotion was analyzed by considering the three emotion groups derived according to Plutchik's emotion classification system. In the recommendation process, data was selected for the location recommendation engine based on these three groups from the assumption that the recommendation should fall on the positive emotion scale. The results of the Tone Analyzer analysis reinforced the use of the positive emotion scale because the collected reviews were more likely to have a positive emotion. Therefore, disgust, fear, anger, and sadness were rearranged into three positive groups (anticipation, joy, and trust) based on Plutchik's comprehensive list of eight primary emotions arranged as opposing pairs (see Table 5). To avoid a negative emotion category, fear and anger were allocated to the joy and trust groups, respectively. Further, we used these three groups to evaluate the influence of user emotion on the recommendation.

In the recommendation process, a prediction of a target user's rating on an unrated target item was calculated by considering the user's rating of observed items. This allows for user-item rating pairs to be used to rate value predictions, as shown in Figure 6 [32].

For item-item collaborative filtering, users who have rated both item $i$ and item $j$ are identified and then the similarities are computed [33]. The similarity calculation is performed based on measures such as the Pearson correlation, Euclidean distance, Tanimoto coefficient, or the loglikelihood similarity. In the proposed process, the similarity
TABLE 5: Emotion groups in the recommendation process.

\begin{tabular}{lcc}
\hline Group I & Anticipation & Anticipation, surprise \\
Group II & Joy & Joy, sadness, fear \\
Group III & Trust & Trust, disgust, anger \\
\hline
\end{tabular}

\begin{tabular}{|c|c|c|c|c|c|c|}
\hline & Item 1 & Item 2 & Item 3 & Item 4 & $\cdots$ & Item $n$ \\
\hline User 1 & 1 & & & 3 & $\cdots$ & \\
\hline User 2 & i & 2 & 5 & i & $\cdots$ & \\
\hline User 3 & 5 & & & 3 & $\cdots$ & \\
\hline User 4 & 1 & 3 & 1 & 1 & $\ldots$ & \\
\hline$\cdots$ & $\cdots$ & $\cdots$ & $\cdots$ & $\cdots$ & $\cdots$ & $\cdots$ \\
\hline User $m$ & 4 & 5 & & 5 & $\ldots$ & \\
\hline
\end{tabular}

FIgURE 6: User-item matrix.

calculation was based on the log-likelihood ratio, which relies on the statistical similarity between two items or users and yielded a sufficient number of items for the recommendation. The log-likelihood ratio utilizes occurrences related to users or items such as users or items that overlap and the events for which both users and items do not have preferences $[34,35]$.

Prediction algorithms estimate the rating that a user would provide for a target item [36]. For item-based prediction, the simple weighted average can be used to predict the ratings [34]. Here, we calculated the predictive rating $P_{u, i}$ by user $u$ for item $i$ as follows:

$$
P_{u, i}=\frac{\sum_{n \in N}(\operatorname{sim}(u, n)+1) \times R_{u, n}}{\sum_{n \in N}(\operatorname{sim}(u, n)+1)},
$$

where $\operatorname{sim}(u, n)$ is the similarity between the $n$th item and user $u$ and $R_{u, n}$ is the rating by user $u$ of item $n$ for all $N$ number of items that are based on the Mahout item-based recommendation algorithm [37]. The similarity calculations 
Sample user behavior actions

\begin{tabular}{|c|c|c|c|c|}
\hline & $S 1$ & $S 2$ & $S 3$ & $S 4$ \\
\hline$U 1$ & 1 & 0 & 1 & 1 \\
\hline$U 2$ & 1 & 1 & 0 & 1 \\
\hline$U 3$ & 1 & 0 & 0 & 1 \\
\hline$U 4$ & 1 & 0 & 0 & 1 \\
\hline
\end{tabular}

FIGURE 7: User-item matrix.

Log-likelihood similarity values
\begin{tabular}{|c|c|c|c|c|}
\hline & $S 1$ & $S 2$ & $S 3$ & $S 4$ \\
\hline U1 & 1 & 0.75 & 0.66 & 0.8 \\
\hline U2 & 0.75 & 1 & 0.5 & 0.6 \\
\hline U3 & 1 & 0.4 & 1 & 0.5 \\
\hline U4 & 1 & 0.5 & 1 & 0.75 \\
\hline
\end{tabular}

FIGURE 8: Similarity values.

ranged from -1.0 to 1.0 , and to avoid negative values, we added 1.0 to similarity values so that the similarity ranges from 0.0 to 2.0. The top-five-places recommendation list was created based on the highest similarity values from the most similar places set from the places pool. Figure 7 illustrates the sample user behavior action for four users, while Figure 8 shows the sample log-likelihood values for four users and four places. Thus, predictive rating calculation is illustrated below by using an example of user-item matrix and similarity values in the recommendation process of user 1 for place $1\left(P_{u 1, s 1}\right)$ and user 1 for place $2\left(P_{u 1, s 2}\right)$.

\subsection{Predictive Rating Calculation (Item-Item Collaboration)}

$$
\begin{aligned}
& P_{u 1, s 1} \\
& =\frac{((1+1) * 1+(1+0.75) * 0+(1+0.66) * 1+(1+0.8) * 1)}{(1+0.75+0.66+0.8)} \\
& =\frac{(2+0+1.66+1.8)}{3.21}=\mathbf{1 . 7} \\
& P_{u 1, s 2} \\
& =\frac{((1+0.75) * 1+(1+1) * 1+(1+0.5) * 0+(1+0.6) * 1)}{(0.75+1+0.5+0.6)} \\
& =\frac{(1.75+2+0+1.6)}{2.85}=\mathbf{1 . 8 8} .
\end{aligned}
$$

Figure 9 shows an example of top-five-places list provided for a user.

3.4. Evaluation. The Travel Destination location recommendation system was presented to 16 users. Each user was asked to evaluate two recommended lists according to the user's preference for each location and the overall preference for the list according to the user's current emotion and overall satisfaction based on the five-point Likert scale. The
TABLE 6: Precision and recall matrix.

\begin{tabular}{lccc}
\hline & Selected & Not selected & Total \\
\hline Relevant & $N_{r s}$ & $N_{r n}$ & $N_{r}$ \\
Not relevant & $N_{i s}$ & $N_{i n}$ & $N_{i}$ \\
\hline Total & $N_{s}$ & $N_{n}$ & $N$ \\
\hline
\end{tabular}

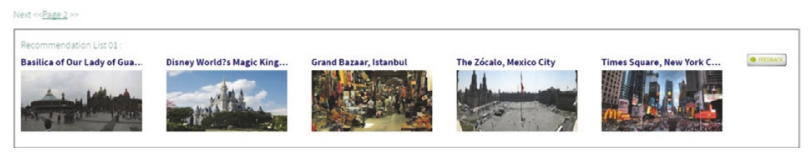

FIGURE 9: Recommendation list.

evaluation was performed to assess the users' opinion of the quality of the CFE recommendation algorithm so that a baseline comparison with the CFN algorithm could be performed.

We used the precision and mean average precision (MAP) values of the two approaches in our evaluation. Also, a $t$ test analysis was performed to test the superiority of CFE against the baseline approach. The $t$-test evaluates $t_{\text {mean }}$ of both average precision (AveP) values and average preference ratings (APR) based on preferred and preferred much user ratings in the five-point Likert scale. Moreover, we evaluated the recommendation list by considering the emotion groups derived at the recommendation engine design stage to track how the lists correspond to user emotions. The overall user satisfaction of the recommendation system was also analyzed. Users were asked to input their emotion from the emoticon scale and evaluate the two lists of five locations.

\section{Results and Discussion}

We used the classification accuracy measure, precision, in our evaluation. This requires a binary d do not recommend/ select, recommend/select\} scale, so we assumed that ratings of 4 and 5 were good recommendations [38].

Based on the precision recall matrix (see Table 6), precision is stated as follows:

$$
\text { precision }=\frac{N_{r s}}{N_{s}} .
$$

The precision values for the CFN and CFE for the 16 users were calculated as below and the mean precision values for the CFE were greater compared with CFN. Average precision calculates the precision at the position of every correct item in the ranked results list of the recommender. The mean of these average precisions across all relevant lists is the mean average precision (MAP). The MAP is also greater for CFE compared to CFN (see Table 7):

$$
\begin{aligned}
& \text { Ave } P=\frac{\sum_{k=1}^{n}(P(k) \times \operatorname{rel}(k))}{\text { number of relevant items }} \\
& \text { MAP }=\frac{\sum_{q=1}^{q} \operatorname{Ave} P(q)}{Q} .
\end{aligned}
$$


TABLE 7: Precision and mean average precision values.

\begin{tabular}{lcc}
\hline Algorithm & Precision \% & Mean av. precision \% \\
\hline CFN & 59.69 & 64.4 \\
CFE & 65.31 & 73.8 \\
\hline
\end{tabular}

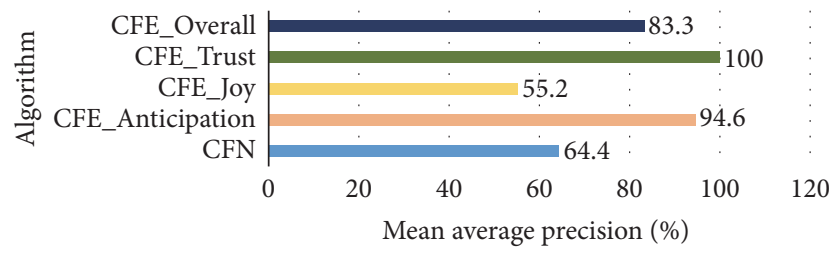

FIGURE 10: Mean average precision values with emotion groups.

In the above, $P(k)$ is the precision at $k$ th element, $\operatorname{rel}(k)$ is 1 if the $i$ th item of the list is relevant, and $Q$ is the total number of lists.

Moreover, we analyzed the mean average precision based on emotional groups (MAPE) for each approach:

$$
\text { MAPE }=\frac{\sum_{c=1}^{C} \sum_{q=1}^{q} \operatorname{Ave} P(q)}{\sum_{c=1}^{C} Q},
$$

where $C$ is the number of emotion groups based upon three groups.

In Figure 10, CFE_Trust, CFE_Joy, CFE_Anitcipation, and CFE_Overall denote the collaborative filtering approach for the trust, joy, and anticipation emotional groups, respectively. We compared the performances of the CF approaches both with and without emotions in terms of average precision values and average preference ratings. The hypotheses are the following:

$H_{0}: \mu_{c}=\mu_{\mathrm{CFN}}$ and alternatively $H_{a}: \mu_{c} \neq \mu_{\mathrm{CFN}}$ and $H_{a}: \mu_{c}>\mu_{\mathrm{CFN}}$, where $\mu_{c}$ and $\mu_{\mathrm{CFN}}$ are the mean average precision rating of the context-aware and noncontext collaborative filtering approaches, respectively.

$H_{0}: \mu_{\mathrm{cp}}=\mu_{\mathrm{CFN}}$ and alternatively $H_{a}: \mu_{\mathrm{cp}} \neq \mu_{\mathrm{CFN}}$ and $H_{a}: \mu_{\mathrm{cp}}>\mu_{\mathrm{CFN}}$, where $\mu_{\mathrm{cp}}$ and $\mu_{\mathrm{CFN}}$ are the mean preference ratings of the context-aware and noncontext collaborative filtering approaches, respectively.

Since $T$ (test statistic) $<t_{\alpha, v}$ (critical value), we reject the null hypothesis in both cases and conclude that the two population means are different at the 0.05 significance level, while for the alternative hypothesis, $\mu_{c}>\mu_{\mathrm{CFN}}$ and $\mu_{\mathrm{cp}}>$ $\mu_{\mathrm{CFN}}$. Therefore, the test results show that the difference with the baseline recommender (CFN) in terms of average precision $(p$ value $=0.509)$ and average rating preferences ( $p$ value $=0.344)$ is statistically significant. Finally, users' feedback on overall satisfaction with the recommended list as well as their opinion based on their current emotion is shown in Table 8 . According to the results, $60 \%$ of users were satisfied overall with the recommended lists. Further, 53.3\% acknowledged that the provided list matched their current emotion.

\section{Conclusion}

In this study, we established how emotion can impact the travel destination recommendation process. The use of emotion as a contextual parameter for location recommendation in conjunction with collaborative filtering increased user satisfaction. In addition, we derived emotion tags for each location based on user reviews to examine how the destination can be effected by emotion in a travel destination recommendation system. While previous studies have incorporated emotion into recommendations for predefined indoor places, our study incorporated it onto a recommendation system for famous tourist attractions. The accuracy of detecting the correct emotion tag using the lexicon-based approach was $63 \%$. However, we believe that this can be improved using other SA approaches.

Plutchik's emotion categorization was used to derive both emotion tags and the acquisition of the emotions of users, and the recommendation list incorporated positive emotion categories. Moreover, the sensitivity of the emotion contextual parameter in the recommendation was analyzed based on the accuracy of the lists for the user.

\section{Discussion and Future Work}

In our approach, we focused on deriving an emotion tag for each destination based on user reviews. Basically, we derived the tags based on Plutchik's emotion categories. The lexiconbased methods for SA are robust, result in good cross-domain performance, and can be easily enhanced with multiple sources of knowledge [39] compared to other approaches.

In deriving emotion tags, opinion mining and other semantic analysis techniques can also be used to enhance accuracy and one such lexical resource, SentiWordNet, which is one dictionary of opinionated terms, is used in such techniques. Also, the deep learning approaches and opining mining techniques, explained in Background, can also be used to enhance the accuracy of emotion word classification. SenticNet is built based on SentiWordNet lexicon and adapts Hourglass of Emotions. In this model, sentiments are reorganized in four independent dimensions that represent different levels of activation. In fact, in this model, affective states are not classified into traditional emotional categories; rather, they are classified into four concomitant but independent dimensions: pleasantness, attention, sensitivity, and aptitude [40].

Although we used exact prefiltering, which for the use of traditional recommendation algorithms does not consider any rating acquired in situations even slightly different from the targeted one, it is proposed that the present system be compared with the context modeling approaches in CARS to allow for an evaluation of the performance of the recommendation engine and that the system be extended to incorporate 
TABLE 8: User satisfaction for top-five-places list.

\begin{tabular}{lcc}
\hline Algorithm & Overall preference for top 5\% & Preference with the emotion of user for top 5 places (\%) \\
\hline CFE & 60 & 53.33 \\
CFN & 46.67 & - \\
\hline
\end{tabular}

user behavior in the system so as to quantitate the sensitivity of each parameter in the recommendation process.

\section{Conflicts of Interest}

The authors declare that there are no conflicts of interest regarding the publication of this paper.

\section{References}

[1] M. Tkalcic, A. Kosir, and J. Tasic, "Affective recommender systems: the role of emotions in recommender systems," in Proc. The RecSys 2011 Workshop on Human Decision Making in Recommender Systems, pp. 9-13, October 2011.

[2] P. Ekman, "Basic Emotions," in Handbook of Cognition and Emotion, pp. 45-60, 1999.

[3] R. Plutchik, "A general psychoevolutionary theory of emotion," Theories of Emotion, vol. 1, no. 4, pp. 3-31, 1980.

[4] L. Castello, Rethinking the meaning of place: conceiving place in architecture-urbanism. Routledge (2016).

[5] G. Debord, Introduction to a critique of urban geography. Critical Geographies A Collection of Readings (1955).

[6] M. Kaminskas and F. Ricci, "Emotion-based matching of music to places," in Emotions and Personality in Personalized Services, pp. 287-310, Springer, 2016.

[7] A. Odic, A. Koir, and M. Tkalcic, "Affective and Personality Corpora," in Emotions and Personality in Personalized Services, pp. 163-178, Springer, 2016.

[8] G. Adomavicius and A. Tuzhilin, "Context-aware recommender systems," in Recommender Systems Handbook, pp. 191-226, Springer US, 2nd edition, 2015.

[9] G. Adomavicius and A. Tuzhilin, "Toward the next generation of recommender systems: a survey of the state-of-the-art and possible extensions," IEEE Transactions on Knowledge and Data Engineering, vol. 17, no. 6, pp. 734-749, 2005.

[10] G. Gonzalez, L. J. De La Rosa, M. Montaner, and S. Delfin, "Embedding emotional context in recommender systems," in Data Engineering Workshop, IEEE 23rd International Conferenc, pp. 845-852, IEEE, 2007.

[11] B. Petrevska and S. Koceski, "Tourism recommendation system: empirical investigation," Revista de turism-studii si cercetari in turism, vol. 14, pp. 11-18, 2012.

[12] M. K. Sarkaleh, M. Mahdavi, and M. Baniardalan, "Designing a tourism recommender system based on location, mobile device and user features in museum," International Journal of Managing Information Technology, vol. 4, no. 2, p. 13, 2012.

[13] Y. Zheng, R. Burke, and B. Mobasher, "Differential context relaxation for context-aware travel recommendation," in International Conference on Electronic Commerce and Web Technologies, pp. 88-99, Berlin, Germany, 2012.

[14] T. De Pessemier, J. Dhondt, K. Vanhecke, and L. Martens, "TravelWithFriends, a hybrid group recommender system for travel destinations," in Workshop on Tourism Recommender
Systems (TouRS15), in Conjunction with the 9th ACM Conference on Recommender Systems (RecSys 2015), pp. 51-60, 2015.

[15] P. Resnick and H. R. Varian, "Recommender systems," Communications of the ACM, vol. 40, no. 3, pp. 56-58, 1997.

[16] K. Goldberg, T. Roeder, D. Gupta, and C. Perkins, "Eigentaste: a constant time collaborative filtering algorithm," Information Retrieval, vol. 4, no. 2, pp. 133-151, 2001.

[17] B. Sarwar, G. Karypis, J. Konstan, and J. Riedl, "Item-based collaborative filtering recommendation algorithms," in Proceedings of the 10th international conference on World Wide Web, pp. 285295, ACM, 2001.

[18] M. Tkalčič, A. Košir, and J. Tasič, “The LDOS-PerAff-1 corpus of facial-expression video clips with affective, personality and user-interaction metadata," Journal on Multimodal User Interfaces, vol. 7, no. 1-2, pp. 143-155, 2013.

[19] G. Adomavicius, R. Sankaranarayanan, S. Sen, and A. Tuzhilin, "Incorporating contextual information in recommender systems using a multidimensional approach," ACM Transactions on Information and System Security, vol. 23, no. 1, pp. 103-145, 2005.

[20] E. Cambria, "Affective Computing and Sentiment Analysis," IEEE Intelligent Systems, vol. 31, no. 2, pp. 102-107, 2016.

[21] W. Medhat, A. Hassan, and H. Korashy, "Sentiment analysis algorithms and applications: a survey," Ain Shams Engineering Journal, vol. 5, no. 4, pp. 1093-1113, 2014.

[22] E. Cambria, S. Poria, R. Bajpai, and B. W. Schuller, "SenticNet 4: a semantic resource for sentiment analysis based on conceptual primitives," in COLING, pp. 2666-2677, 2016.

[23] M. M. Lopez and J. Kalita, "Deep Learning applied to NLP," https://arxiv.org/abs/1703.03091.

[24] O. Araque, I. Corcuera-Platas, J. F. Sánchez-Rada, and C. A. Iglesias, "Enhancing deep learning sentiment analysis with ensemble techniques in social applications," Expert Systems with Applications, vol. 77, pp. 236-246, 2017.

[25] S. Poria, E. Cambria, and A. Gelbukh, "Deep convolutional neural network textual features and multiple kernel learning for utterance-level multimodal sentiment analysis," in Proceedings of the Conference on Empirical Methods in Natural Language Processing, EMNLP 2015, pp. 2539-2544, prt, September 2015.

[26] Y. Zheng, "Adapt to Emotional Reactions In Context-aware Personalization," in Proceeding of the 4th Workshop on Emotions and Personality in Personalized Systems (EMPIRE), p. 1, 2016.

[27] S. M. Mohammad and P. D. Turney, "Crowdsourcing a wordemotion association lexicon," Computational Intelligence, vol. 29, no. 3, pp. 436-465, 2013.

[28] Y. H. Hu, P. J. Lee, K. Chen, J. M. Tarn, and D. V. Dang, "Hotel Recommendation System based on Review and Context Information: a Collaborative filtering Appro," in PACIS, p. 221, 2016.

[29] , The Macquarie Thesaurus. Macquarie Library, J. Bernard, Ed., Sydney, Australia, 1986.

[30] S. Aman and S. Szpakowicz, "Identifying expressions of emotion in text," in International Conference on Text, Speech and Dialogue, pp. 196-205, Berlin, Germany, 2007. 
[31] H. Tang, S. Tan, and X. Cheng, "A survey on sentiment detection of reviews," Expert Systems with Applications, vol. 36, no. 7, pp. 10760-10773, 2009.

[32] S. Gong, "A collaborative filtering recommendation algorithm based on user clustering and item clustering," Journal of Software, vol. 5, no. 7, pp. 745-752, 2010.

[33] X. Su and T. M. Khoshgoftaar, "A survey of collaborative filtering techniques," Advances in Artificial Intelligence, vol. 4, 2009.

[34] T. Dunning, "Accurate methods for the statistics of surprise and coincidence," Computational Linguistics, vol. 19, no. 1, pp. 61-74, 1993.

[35] M.-Y. Hsieh, W.-K. Chou, and K.-C. Li, "Building a mobile movie recommendation service by user rating and APP usage with linked data on Hadoop," Multimedia Tools and Applications, vol. 76, no. 3, pp. 3383-3401, 2017.

[36] M. Papagelis and D. Plexousakis, "Qualitative analysis of userbased and item-based prediction algorithms for recommendation agents," Engineering Applications of Artificial Intelligence, vol. 18, no. 7, pp. 781-789, 2005.

[37] S. Schelter and S. Owen, "Collaborative filtering with apache mahout," in Proceedings of the ACM RecSys Challenge, 2012.

[38] G. Shani and A. Gunawardana, "Evaluating recommendation systems," Recommender Systems Handbook, pp. 257-297, 2011.

[39] M. Taboada, J. Brooke, M. Tofiloski, K. Voll, and M. Stede, "Lexicon-based methods for sentiment analysis," Computational Linguistics, vol. 37, no. 2, pp. 267-307, 2011.

[40] E. Cambria, R. Speer, C. Havasi, and A. Hussain, "SenticNet: A publicly available semantic resource for opinion mining," in Proceedings of the 2010 AAAI Fall Symposium, pp. 14-18, November 2010. 

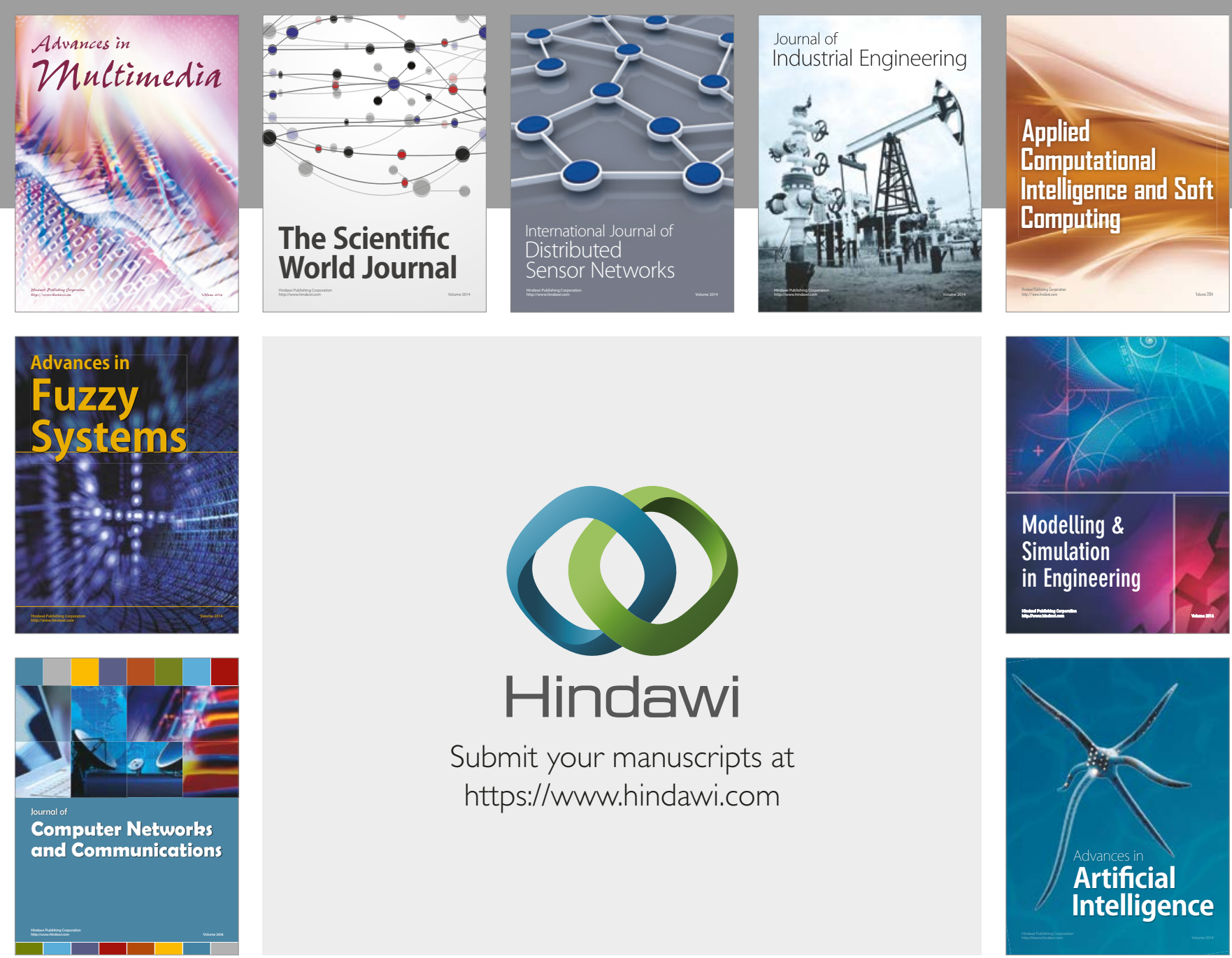

\section{Hindawi}

Submit your manuscripts at

https://www.hindawi.com
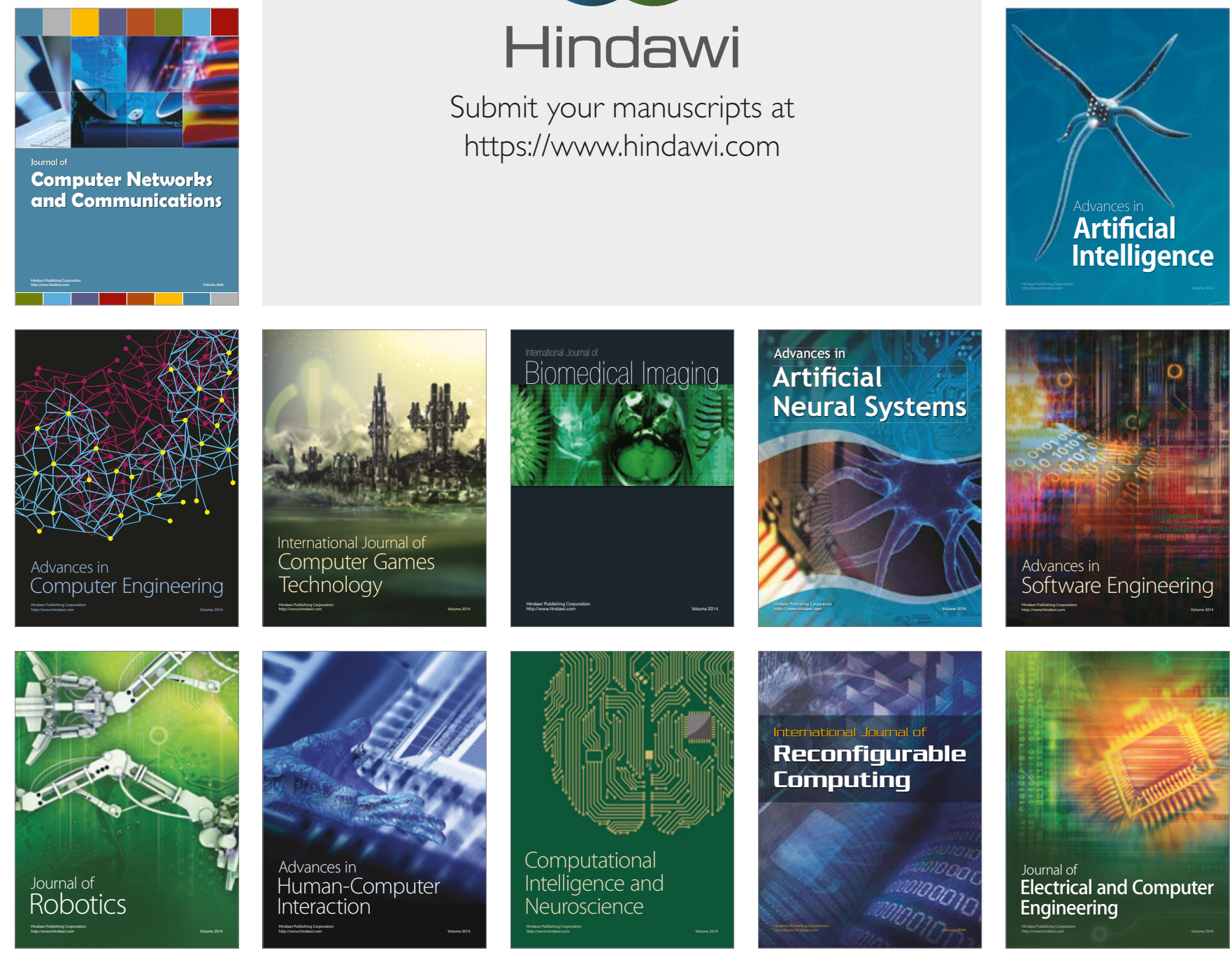